\title{
APPLICATIONS AND PHYSICS OF THE TABLETOP STORAGE RING
}

\author{
H. Yamada*, Y. Kitazawa ${ }^{1}$, I. Tohyama ${ }^{1}$, N. Takeichi, T. Takayama ${ }^{2}$, K. Ozaki, Y. Sakai, T. \\ Kaneda, and K. Saka, Ritsumeikan, Shiga, A.I. Kleev, G.D.Bogomolov, V. Zavialov, Kapitza Inst., \\ Moscow, H. Hama, M. Hosaka, J. Yamazaki, UVSOR, Okazaki, T. Koseki, H. Takaki, ISSP, Tokyo
}

\begin{abstract}
We are constructing the smallest electron storage ring for establishing hard/infrared/far-infrared beam facility. Physics subject to be studied are the study on a $2 / 3$ resonance injection; the sub-pico second short electron bunch formation and the coherent synchrotron radiation; the photon storage ring (PhSR) lasing mechanism, the light pulse compression in the PhSR; the Compton backscattering under strong magnetic field; and Characteristics of hard x-rays generated by a very thin wire target placed in the orbit.
\end{abstract}

\section{HISTORY AND PRESENT STATUS}

We are constructing the smallest electron storage ring for establishing hard x-ray as well as infrared/far-infrared beam facility [1-3]. This construction was initially motivated by an idea of the photon storage ring (PhSR)[47], which is a novel laser scheme originated by Yamada in 1989. The physics involved in the PhSR is similar to a free-electron laser, but an undulator is not used. Instead, one barrel shaped mirror provides the function of the undulator as well as an optical cavity in corroborating with a bunched electron beam circulating in an exact circular orbit. The hard x-ray generation scheme was later added to the project according to the Yamada's proposal in 1996 [8].

The storage ring was designed in 1993, funded by Ministry of Education in 1994, completed in 1997 (a 1 $\mathrm{kW}$ magnetron instead of klystron was used because of financial limit), and placed in the injector room of UVSOR Okazaki. We have performed $2 / 3$ resonance injection by using a $15 \mathrm{MeV}$ linac. We have observed circulating beams by a loss monitor. It was impossible to optimise injection and to store useful amount of current since the characteristic wavelength of synchrotron radiation is too long to monitor by fast response detector and another monitors was unavailable in the strong magnetic field of the ring.

A radiation safety room was built in 1996 at Ritsumeikan University, and the ring was moved from

*1-1-1Nojihigashi, Kusatsu, Shiga, Japan;

e-mail: hironari@ se.ritsumei.ac.jp;

Work supported in part by the Science and Technology Corp. of Japan

On leave from the Photon Production Laboratory Ltd.

${ }^{2}$ Sumitomo Heavy Industries Ltd.
Okazaki in 1998. A cw klystron was funded in 1997. Science and Technology Corp. of Japan funded a 21.5 $\mathrm{MeV}$ microtron in 1998. The microtron was built in collaboration with a team from P.L.Kapitza institute for physical problems, and succeeded in the commissioning last fall.

In order to adapt the resonance injection at $21.5 \mathrm{MeV}$, we have modified magnetic field distribution by adjusting pole shape with thin circular metal plates. Parameters of the smallest ring system are shown in Table 1.

FIG. 1. An overview of the tabletop electron storage ring system under construction installed in the $2 \mathrm{~m}$ deep and $3 \times 7 \mathrm{~m}^{2}$ wide pit.

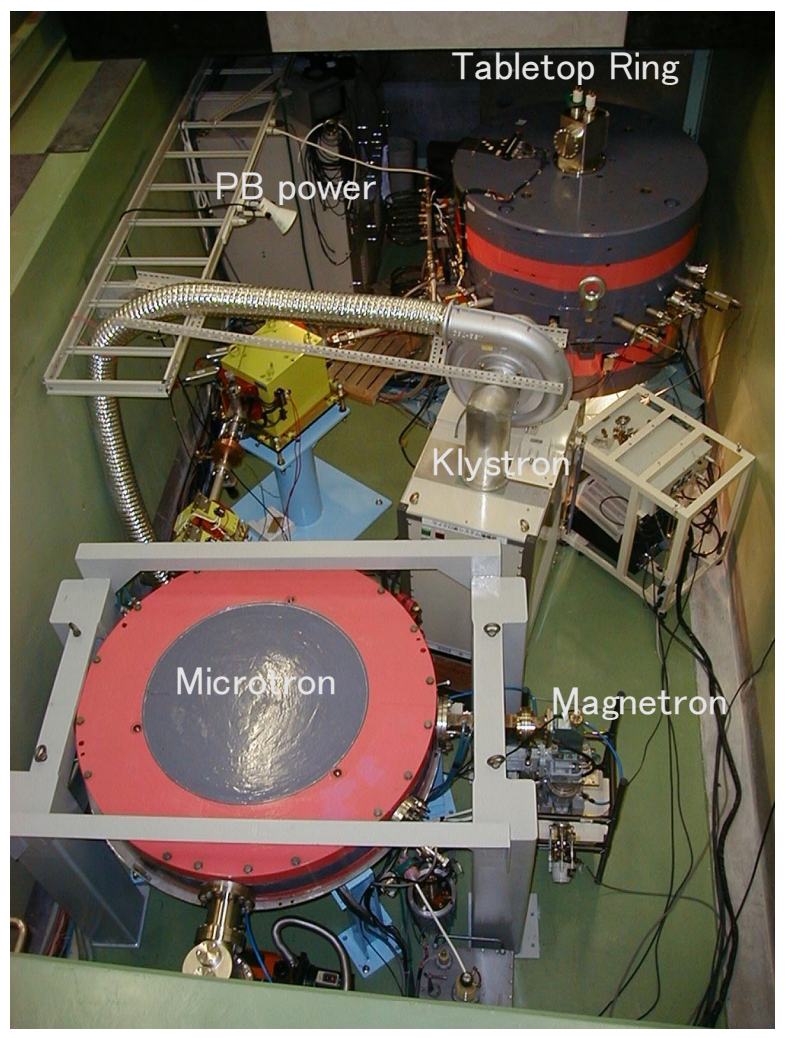

\section{CONFIGURATION OF THE SYSTEM}

The smallest ring system is made of a $1.2 \mathrm{~m}$ out diameter and $1.5 \mathrm{~m}$ high normal conducting magnet and the circular microtron injector of $1.2 \mathrm{~m}$ out diameter as 


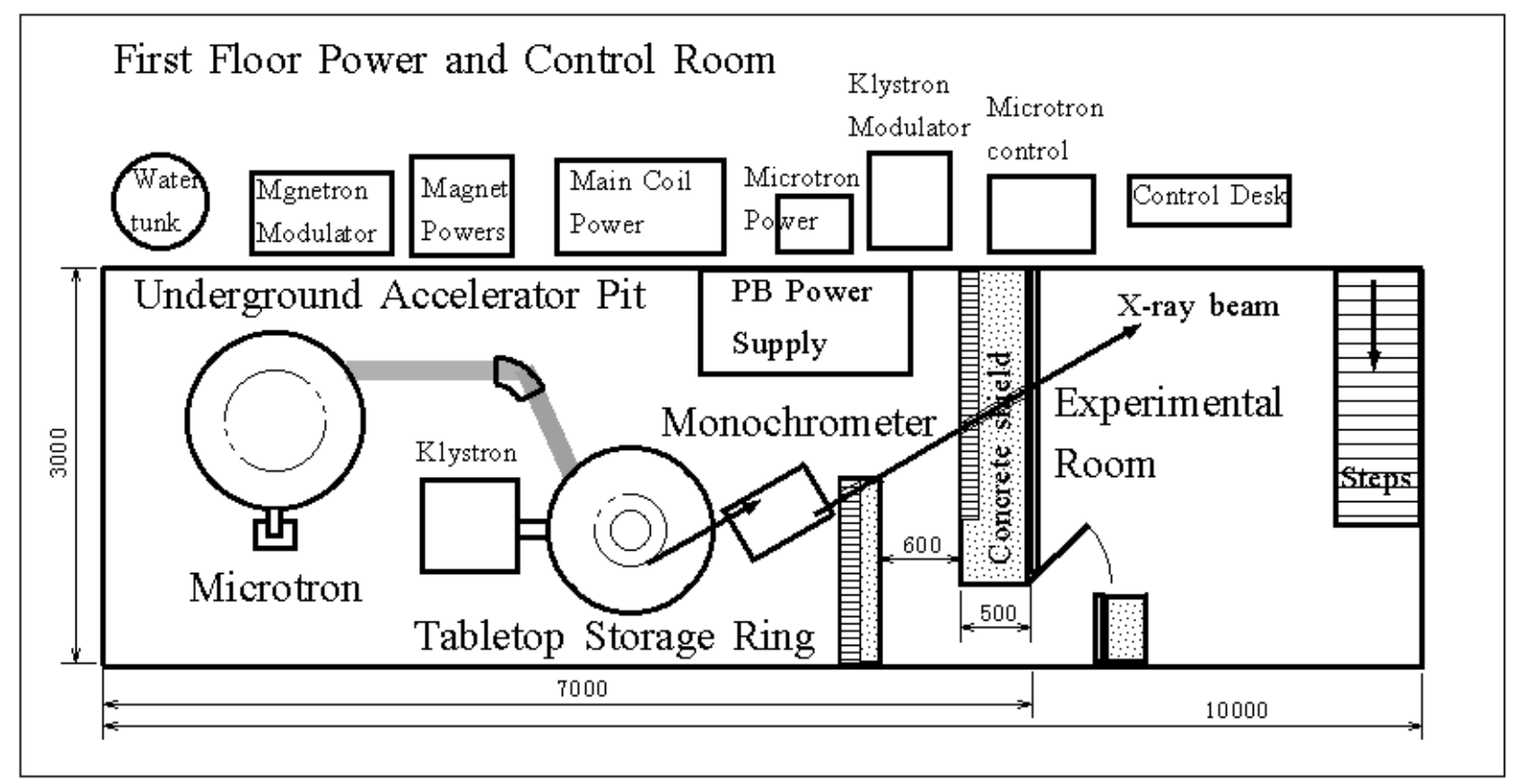

FIG. 2. A schematic plane view of the facility is shown. A small experimental room is seen which can be utilised for x-ray beam applications. A double crystal monochrometer is placed inside the accelerator room

seen in Fig. 1. The ring is an exact circular type using one piece of weak focusing magnet. The magnet weigh is 8 ton in total to store electrons up to $50 \mathrm{MeV}$. The system is placed in a $7 \times 3 \mathrm{~m}^{2}$ wide and $2 \mathrm{~m}$ deep pit as seen in Fig. 2 and is covered with $0.5 \mathrm{~m}$ thick concrete blocks. A small exposure room is attached. The x-ray as well as infrared/farinfrared beams can be extracted through a channel in the wall and through a double crystal monochrometer system (the crystal can be replaced to spherical mirrors for IR and FIR) placed inside the accelerator room to stop energetic $\gamma$ rays.

\section{APPLICATIONS AND PHYSICS PROBLEMS}

Beam injection is scheduled from April 29. We are ready to study the following physics related to the smallest ring, and several beam application programs for initial demonstration.

Physics subject involved in the smallest electron storage ring are the following:

1. Experimental study on $2 / 3$ resonance injection method.[2]

2. Beam instability problems appears in the lowest energy ring.[2]

3. Study on a forced cooling technique by laser and residual ions [9].

4. Sub-pico second short electron bunch formation [10].
Table 1 Main specifications of the system

\begin{tabular}{|r|c|}
\hline \multicolumn{1}{|l|}{ Magnet } & $\begin{array}{c}\text { Weak focusing, normal } \\
\text { conducting, Cylindrical }\end{array}$ \\
\hline Pole gap & $100 \mathrm{~mm}$ \\
\hline Orbit radius & $0.156 \mathrm{~m}$ \\
\hline n-value & 0.52 \\
\hline RF Cavity frequency & Two set, re-entrant type \\
\hline Harmonics & $2.44 \mathrm{GHz}$ \\
\hline RF-voltage & 8 \\
\hline RF Source & Magnetron \\
\hline Perturbator & Pair of one turn coils \\
\hline pulse source & $0.4 \mu \mathrm{s}$ width, $6500 \mathrm{~A}$ peak current \\
\hline Repitition rate & Max $100 \mathrm{~Hz}$ \\
\hline Injector & $21.5 \mathrm{MeV} \mathrm{microtron}$ \\
\hline Critical wavelength & $8.1 \mu \mathrm{m}$ \\
\hline Damping time & $1.0 \mathrm{~s}$ \\
\hline
\end{tabular}

5. Coherent synchrotron radiation measurement.

6. Photon storage ring lasing mechanism [7]. Effect of the coherent radiation on the laser gain [6].

7. Light pulse compression in the photon storage ring and femt-second pulse formation [11].

8. Compton back-scattering under strong magnetic field [12].

9. Characteristics of hard $x$-ray beam generated by the very thin target placed in the orbit [8].

The 7-th subject is added according to the recent 
theoretical study on the PhSR. The PhSR theory was earlier constructed on an assumption that a single mode electro-magnetic(EM) field (whispering garelly mode) appears in the circular optical cavity [7]. As a consequence the laser gain is calculted to the interaction between uniformly filed EM field and electron bunches. Recently we have improved the model and mathematical formalism [11]. We represent the mode in superposition of the multiple radial modes, and obtain the comprex wave numbers and each amplitude coefficient by solving eigen functions, which are determined by boundary conditions at optical cavity mirror surface and the envelop of circulating electron beam. If the bunched electron is not taken into account, the uniform EM field appears. We found that when the ratio between the mirror radius and the electron orbit radius sutisfyes the Yamada's resonance condition, the EM field becomes pulse mode. It will happen that the optical pulse width is narrower than the electron bunch width when the peak current is high and the mirror/orbit ratio is close to the resonance value as seen in FIG. 3.

In the next we discuss the primary beam applications, which are;

1. X-ray imaging using the phase-contrast method.

2. Medical treatment of arterioscleroses, cancer, lighiasis etc. by FIR beam [1].

3. A bio-molecular dynamics excited by FIR-rays [1].

4. Analysis of the water network structure effected by biomolecules.

In our system the x-ray emission mechanism is not the synchrotron radiation but the bremsstrahlung from a thin wire target placed in the electron orbit [].

Regardless of magnetic or electric forces, the emission occurs due to acceleration of electrons. Characteristics of the photon emission are simply determined by the energy and momentum conservation lows. The bremsstrahlung should be emitted in the strongly forward direction in a $1 / \gamma$ (the $\gamma$ is the Lorentz factor) cone as same as the synchrotron radiation when the electron energy is relativistic. The high- energy $\mathrm{x}$-ray can be generated more easily by the bremsstrahlung than by the synchrotron radiation, since the atomic Coulomb force is much stronger at the vicinity of nucleus than the magnetic field of bending magnet.

The characteristics of this $x$-ray source are quite different from that of synchrotron light sources in many regards. First of all, the beam lifetime is only minutes therefor we keep beam injection continuously. But because of the resonance injection, the beam in the central orbit will never be disturbed by the injection. Thus we will be able to keep the constant beam intensity. In the second, the x-ray spectral brightness is rather flat and is extended to the high energy up to the electron energy. The monochrometer is an essential need. We can select any x-ray energy band according to an aim of exposure. In the third we can obtain source size as small as that of Spring-8, since that is determined by the target size as $10 \mu \mathrm{m}$ in our case. Beam position must be very stable since the source point is fixed. This is often the problem in the case of large synchrotrons due to sunshine and moon. The $\mathrm{x}$-ray beam covers wide angle such as $1 / \gamma=$ $0.01 \mathrm{rad}$, since the electron energy is low. At $1 \mathrm{~m}$ distant from the source point the $10 \times 10 \mathrm{~mm}^{2}$ area can be exposed at a time. These all features are advantageous for the phase contrast $\mathrm{x}$-ray imaging.

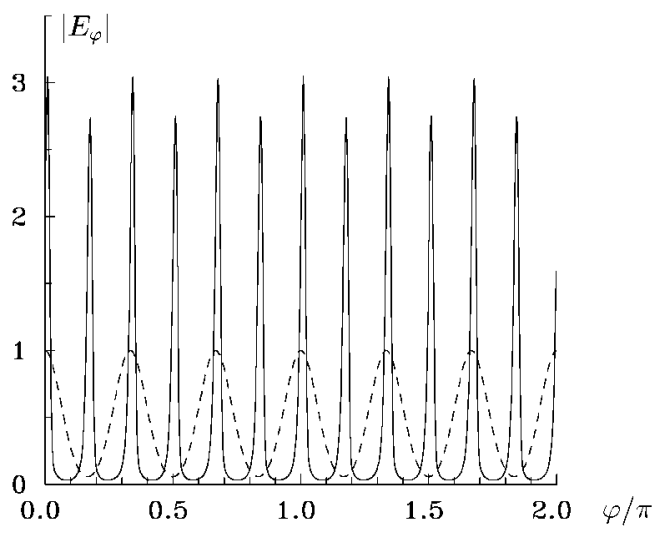

FIG. 3. Light pulse is compressed in the circular optical cavity, when the bunched beam exists. The dashed line represent the electron bunches, and the sold the optical pulse.

\section{REFERENCES}

[1] H. Yamada, J. Synchrotron Rad. (1998), 5, 1326-1331

[2] H. Yamada, Advances in Colloid and Interface Sci. 71-72, (1997) 371-392.

[3] H. Yamada et.al., Proc. $1^{\text {st }}$ Asian Particle Accelerator Con. Tsukuba, KEK, Mar 6, 1998, www-proc.kek.jp/proc.html.

[4] H.Yamada, Japanese J. Appl. Phys. 28(9) (1989) L1665; H.Yamada, Nucl. Instrum. Methods in Phys. Res. A304 (1991) 700-702.

[5] H. Yamada, Nucl. Instrum. Methods in Phys. Res. B79, (1993) $762-766$

[6] H. Yamada, H. Tsutsui, K. Shimoda, and K. Mima, Nucl. Instrum. Methods. in Phys. Res. A331 (1993) 566-571

[7] Mima K., Shimoda K., and Yamada H., IEEE J. Quantum Electronics, 27, (1991) 2572-2577.

[8] H. Yamada, Jpn. J. Appl. Phys., 35 (1996) L182-L185.

[9] H. Yamada to be published.

[10] H. Yamada , AIP CONFERENCE PROC. 367, (1996) 165-180.

[11] A.I. Kleev and H. Yamada, Nucl. Instrum. Methods in Phys. Res

[12] I. Sakai and H. Yamada, Proceeding of $11^{\text {th }}$ Conference on Accelerator Science and Technology, Himeji,Japan, 1998. 